\title{
Losa curva de hormigón armado para Centro de Convenciones
}

\author{
Reinforced concrete curve slab for Conventions Center
}

Fecha de entrega: 16 de octubre 2020

Fecha de aceptación: 12 de abril 2021

\section{Orlando Reyes ${ }^{1}$, Isel del Carmen Díaz ${ }^{1}$, Annabel Ramos² y Janet Otmara Martínez ${ }^{1}$}

${ }^{1}$ Departamento de Estructuras, Facultad de Ingeniería Civil, Universidad Tecnológica de La Habana José Antonio Echeverría CUJAE, Calle 114 no. 11901 entre Ciclovía y Rotonda, Municipio Marianao, CP 19390, La Habana, Cuba, oreyesv@civil.cujae.edu.cu, iselcarmen@civil.cujae.edu.cu, jcid@civil.cujae.edu.cu (ORCID: 0000-0003-1969-1553)

${ }^{2}$ Empresa de Proyectos de Arquitectura e Ingeniería de La Habana EPROB, Calle Goss 677 entre Amado y Aranguren, Municipio Diez de Octubre, La Habana, Cuba, aramos@eprob.cu

En la actualidad se desarrollan novedosos proyectos de obras que implican consideraciones adicionales en la modelación, el análisis y el diseño estructural. Un ejemplo de esta afirmación lo constituyen las losas estructurales con formas complejas, de amplio empleo en las propuestas arquitectónicas contemporáneas. Este trabajo tiene el objetivo de presentar la concepción estructural, la modelación, el análisis y el diseño de la losa de cubierta curva del Centro de Convenciones de un hotel ubicado en La Habana. Para la modelación se empleó el programa ETABS v17. El diseño incluye el cálculo del refuerzo para el Estado Límite Último y la verificación del Estado Límite de Servicio de deformación y fisuración según ACI-318 (2019) y NC-207 (2019). Se verifica, además, el cumplimiento de los requisitos de resistencia al fuego.

Palabras clave: losa curva, MEF, ETABS, losa de hormigón armado, fisuración, tiempo de resistencia al fuego
At present, new building projects are being developed that involve additional considerations in computational modelling, analysis and structural design. An example of this is the structural slabs with complex shapes, widely used in contemporary architectural proposal. This work has the objective of presenting the structural conception, computational modelling, analysis and structural design of the curve roof slab of the Convention Center of a hotel located in Havana. ETABS v17 software was used. The analysis includes the calculation of reinforcement based on the Strength Limit and the verification of Serviceability in terms of deflections and cracking by ACI-318 (2019) and NC-207 (2019). Compliance with fire resistance requirements is also verified.

Keywords: curve slab, FEM, ETABS, reinforced concrete slab, cracking, fire resistance time

\section{Introducción}

Las losas constituyen elementos estructurales cuya función principal radica en resolver el sistema horizontal de una edificación, siendo utilizadas fundamentalmente en las soluciones de entrepisos y cubiertas (Hernández y Hernández, 2010). Las mismas han sido definidas de forma clásica como elementos estructurales planos o superficiales limitados por dos planos paralelos en los que predominan dos dimensiones frente a la tercera, y sobre los cuales las cargas actúan, básicamente, perpendicularmente a su plano medio, incluyendo el peso propio. Este tipo de elemento estructural se encuentra sometido fundamentalmente a esfuerzos de flexión en una o dos direcciones (ACI-318,
2019; González y Robles, 2005; NC-207, 2019).

Las losas o placas pueden diferenciarse por su forma, por la disposición y tipo de los apoyos y por la condición de borde de estos. Lo anterior, unido a la variedad de tipos de cargas que sobre estas pueden actuar, hace que sean numerosos y en ocasiones complejos los problemas a resolver en el análisis de las mismas (Gherbi et al., 2018).

Para el análisis de losas existen dos grupos de métodos, los métodos clásicos, que se basan en la teoría de la elasticidad que suponen que el material es homogéneo e isótropo y se comporta linealmente; y los métodos en rotura basados en la teoría de la plasticidad que suponen al material como 
rígido-plástico perfecto. Con los métodos clásicos pueden obtenerse, con buena aproximación, los esfuerzos en la situación de servicio y con los métodos de rotura, la carga última de agotamiento (Jiménez et al., 2000).

La modelación computacional basada en el Método de Elementos Finitos (MEF) es una eficaz herramienta para la simulación y representación de fenómenos diversos en ingeniería, entre los que se encuentran el análisis del comportamiento de losas. Se han desarrollado modelos para el estudio del efecto del refuerzo por punzonamiento, la determinación del patrón de fallo así como el comportamiento por deformación bajo diferentes condiciones de borde y carga con resultados satisfactorios (Genikomsou y Polak, 2017; Gherbi et al., 2018; Rasoul y Taher, 2019).

En el litoral oeste de la ciudad de La Habana, se pretende realizar la construcción de un Hotel de Negocios y Ocio. El hotel se compone de cuatro partes funcionales principales: el centro del vestíbulo de entrada común (Lobby), el Hotel de Negocios, el Hotel de Ocio y el Centro de Convenciones. El Centro de Convenciones se caracteriza por poseer una losa de cubierta curva de grandes dimensiones (Figura 1).

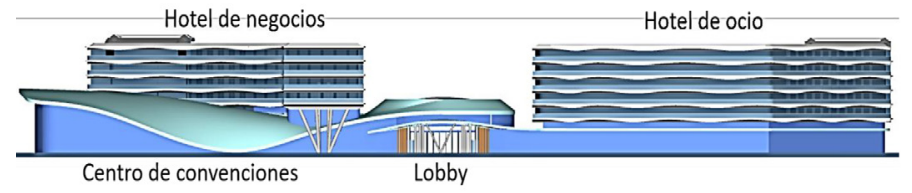

Figura 1: Elevación frontal del hotel donde se aprecian las cuatro zonas funcionales principales y la forma de la losa del Centro de Convenciones.

La forma de la losa y las cargas asociadas a la misma entre las que se encuentra la carga de viento dada la ubicación de la edificación, obligan a la necesidad de realizar el análisis mediante el empleo de la modelación computacional con el Método de los Elementos Finitos MEF.

En el presente trabajo se describe el modelo de la losa realizado con el empleo del programa ETABS v17 (CSI, 2017), el análisis del comportamiento de la misma y el diseño estructural que considera los Estados Límites de Servicio y Últimos así como la verificación de requisitos de resistencia al fuego. Se brindan, además recomendaciones para su ejecución.

\section{Premisas del proyecto}

El proyecto del hotel en su diseño y ejecución, deberá corresponder a las circunstancias del entorno tales como:

- La preponderancia del mar a partir de la consideración del potencial que brinda su uso para actividades náuticas y de playa, así como la alta incidencia de este en la imagen del conjunto.

- Su localización en la entrada de un canal de la marina, hace que se trate de un conjunto de alta significación, en su funcionalidad e imagen expresiva.

- El carácter rector de la declaración de Zona de Protección a la zona de valor histórico-cultural donde se ubica, el factor de riesgo medioambiental y la vulnerabilidad de la zona, al estar ubicada en zona primaria de riesgo por penetración del mar, además de las afectaciones previstas por el cambio climático.

El hotel estará ubicado en la costa a menos de $50 \mathrm{~m}$ del mar. El terreno es llano con una cota promedio en la parcela de $+1.5 \mathrm{~m}$ sobre el nivel del mar y como cota de inundación $+2.80 \mathrm{~m}$. Basado en esa información se decide fijar como \pm 0.00 la cota de +5.40 m equivalente a $2.60 \mathrm{~m}$ por encima del nivel de inundación máximo de $+2.80 \mathrm{~m}$.

\section{Centro de convenciones}

El Centro de Convenciones abarca un área total de 3837.70 $\mathrm{m}^{2}$ aproximadamente. El largo máximo es de $79.22 \mathrm{~m}$ desde el borde exterior hasta la junta de expansión ubicada antes del Hotel de Negocios mientras que el ancho mayor es de 68.15 m (Figuras 2a,b). La losa de cubierta, de hormigón armado, tiene $200 \mathrm{~mm}$ de espesor y un área aproximada de $4008,20 \mathrm{~m}^{2}$. Esta se caracteriza por tener una forma geométrica curva, sin simetría longitudinal ni transversal (Figuras 2c,d,e).

El Centro estará compuesto por múltiples locales entre los cuales se encuentran almacenes, centros de negocios, cocina, salones independientes para conferencias, un auditorio con capacidad para 400 asientos y una sala multifunción con capacidad para 800 asientos. La solución estructural concebida fue a partir de pórticos de hormigón armado dispuestos en dos direcciones. 


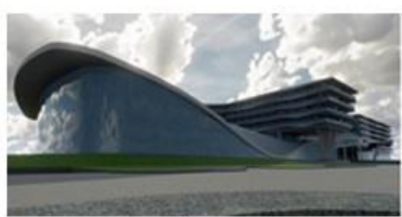

(a)

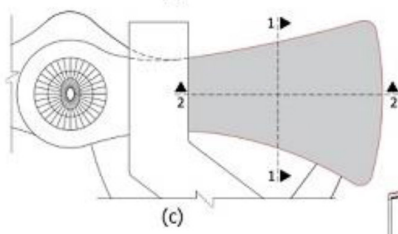

(c)
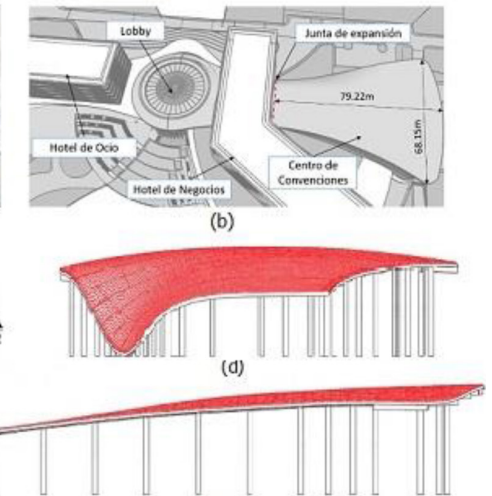

(e)
Figura 2: Geometría de la losa del Centro de Convenciones. a) Isométrico, b) ubicación de la junta de expansión, c) vista en planta, d) corte 1-1 y e) corte 2-2

\section{Modelación computacional}

El proceso de modelación fue desarrollado con el empleo del programa ETABS v17 (CSI, 2017). La geometría de la losa fue representada en el programa SketchUp y exportada para el posterior trabajo en ETABS.

\section{Geometría}

La estructura presenta un total de 53 columnas de hormigón armado, distribuidas en: 18 con sección circular de $800 \mathrm{~mm}$ de diámetro, 20 de sección cuadrada de $800 \mathrm{~mm}$ de lado y 15 de sección rectangular con dimensiones variables. Las longitudes de estas columnas oscilan entre los $4.58 \mathrm{~m}$ y $15.94 \mathrm{~m}$. En el caso de las vigas, hay un total de 141 vigas curvas, dispuestas en vigas principales y secundarias. Se destacan las del salón multifunción y del auditorio.

La sección de las vigas principales es de $0.70 \mathrm{~m}$ x 2.00 $\mathrm{m}$ para una luz de $28.17 \mathrm{~m}$ y $0.60 \mathrm{~m}$ x $1.60 \mathrm{~m}$ para una luz de $18.79 \mathrm{~m}$, en el salón multifunción y el auditorio respectivamente. Las vigas secundarias son de $0.35 \mathrm{~m} \mathrm{x}$ $0.70 \mathrm{~m}$ para una luz de aproximadamente $8.40 \mathrm{~m}$, en todos los locales (Figura 3).

\section{Materiales}

La agresividad del medio influye directamente en la durabilidad de la estructura. En este aspecto tiene un gran impacto la relación agua/material cementante $(\mathrm{a} / \mathrm{mc})$ y la composición de los materiales cementantes utilizados en el hormigón. Debido a la dificultad que supone verificar, una vez construido el elemento estructural, la relación a/mc del hormigón, el valor tomado de la resistencia característica

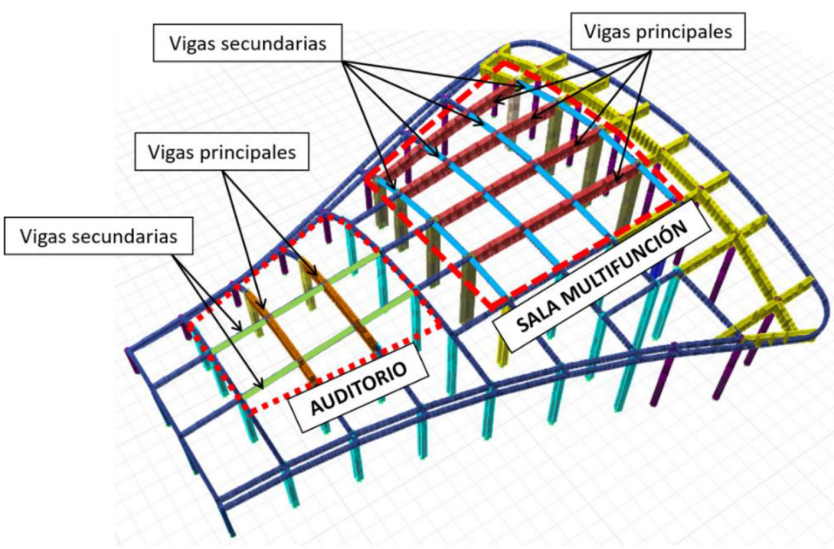

Figura 3: Distribución de vigas y columnas del Centro de Convenciones

a compresión a los 28 días $\left(f_{c}^{\prime}\right)$ debe ser congruente con la máxima relación a/mc requerida por los efectos de durabilidad. La selección de una resistencia a compresión adecuada $f_{c}^{\prime}$, permite que se puedan utilizar los resultados de ensayos de resistencia como un substitutivo de a/mc, y así ayudar a evitar que se exceda en obra la máxima a/mc. Los requisitos para un $f_{c}^{\prime}$ mínimo se utilizan para garantizar que se produzca una pasta de cemento de alta calidad (ACI-318, 2019).

La edificación se ubica en una zona costera a menos de 50 $\mathrm{m}$ del mar por lo que la agresividad del ambiente clasifica como muy alta (NC-250, 2005; NC-207, 2019). ACI-318 (2019) clasifica este tipo de exposición ambiental dentro de la Categoría de Exposición C, referida a hormigón expuesto a condiciones que requieren protección adicional del refuerzo contra la corrosión, y dentro de esta categoría clasifica como clase $\mathrm{C} 2$, que es la referente a hormigón expuesto a la humedad y a una fuente externa de cloruros provenientes de agua de mar o salpicaduras del mismo origen. En el caso de la resistencia característica a compresión del hormigón a los 28 días $f_{c}^{\prime}$, NC-250 (2005) y NC-207 (2019) establecen para la categoría de exposición antes mencionada una resistencia a compresión mínima de $30 \mathrm{MPa}$ y la máxima relación agua/cemento de 0.40 . Se estableció, por tanto, $f_{c}^{\prime}=30 \mathrm{MPa}$, siendo el módulo de deformación $\left(E_{c}^{\prime}\right)$ de $25740 \mathrm{MPa}$ utilizados en la modelación, el análisis y el diseño estructural.

Existen numerosas expresiones matemáticas para modelar el comportamiento del hormigón a compresión uniaxial (e.g. Salguero et al., 2013). Como hipótesis básica de 
los diseños a flexión se establece que la relación entre la distribución de esfuerzos del hormigón en compresión y su deformación, se puede suponer que es rectangular, trapezoidal, parabólica (Figura 4a) o de cualquier otra forma que resulte de la predicción de la resistencia y que coincida con resultados de extensos ensayos (NC-207, 2019). Se empleó un diagrama rectangular equivalente con bloque de compresión de ancho constante $\alpha f_{c}^{\prime}$ y profundidad $\beta_{1} c$, siendo $\alpha=0.85$ y $\beta_{1}$ función de la resistencia a compresión del hormigón, ambos independientes de la ley constitutiva que se adopte (ACI-318, 2019; NC-207, 2019). La distancia $c$ medida desde la fibra de deformación unitaria máxima hasta el eje neutro, se evalúa en dirección perpendicular a dicho eje.

El acero utilizado como refuerzo del hormigón es de calidad G-60, con una tensión de fluencia $f_{y}$ de $420 \mathrm{MPa}$ y módulo de deformación $E_{s}$ de $2 \cdot 10^{5} \mathrm{MPa}$ (ACI-318, 2019; Hernández y Hernández, 2010; McCormac y Brown, 2011; NC-207, 2019). En el caso del diagrama tensióndeformación unitaria del acero, se adopta el diagrama bilineal con escalón de fluencia como se muestra en la Figura 4b (NC-207, 2019).
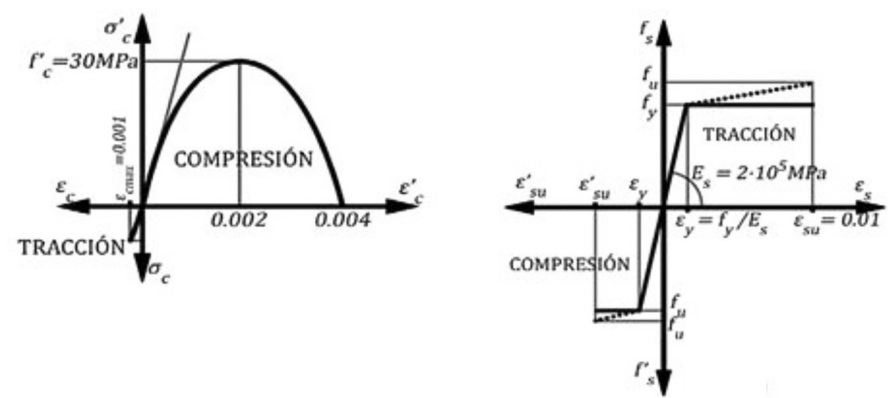

Figura 4: Leyes constitutivas de los materiales: a) hormigón y b) acero (Hernández y Hernández, 2010)

La agresividad del medio impone también un valor de recubrimiento neto para los elementos estructurales, considerando para la losa $40 \mathrm{~mm}$ y para las vigas $50 \mathrm{~mm}$ (NC-207, 2019; NC-250, 2005).

\section{Condiciones de borde}

Los pórticos de soporte de la losa fueron considerados continuos en la unión viga - columna y las columnas empotradas en la base. En la modelación de la losa, la unión entre los paños en los que fue dividida se consideró continua.

\section{Cargas}

Fueron consideradas las cargas permanentes, de uso y ambiental de viento. En las cargas permanentes además del peso propio de los elementos se consideró una sobrecarga equivalente a $3.21 \mathrm{kN} / \mathrm{m}^{2}$ que incluye el peso del mortero de nivelación, sistema de impermeabilización, falso techo, carga de instalaciones y terminación de cristalería sobre la cubierta (NC-283 2003). Como carga de utilización fue considerada $0.8 \mathrm{kN} / \mathrm{m}^{2} \mathrm{NC}-284$ (2003). Para la determinación de la carga ambiental de viento fue adoptado el procedimiento establecido en la NC-285 (2003). Esta normativa establece la consideración de un análisis estático cuando el período de oscilación de la estructura $T$ es menor que un segundo $(T<1 \mathrm{~s})$. En el Centro de Convenciones se realizó un análisis estático debido a que el periodo fundamental de oscilación obtenido es de 0.91 $\mathrm{s}$, considerando como masa el efecto correspondiente a $D+0.5 L$, siendo $D$ la carga permanente y $L$ la carga de utilización. La determinación de la componente estática se realizó a partir de la expresión (1):

$q_{\text {extremo }}=q_{10}$ Ct Cs Cr Ch Cra Cf

donde $q_{\text {extremo }}$ es la carga de viento extremo en $\mathrm{kN} / \mathrm{m}^{2}$, es la presión básica del viento y $C t, C s, C r, C h, C r a, C f$, son los coeficientes de recurrencia, de sitio, de ráfaga, de altura, de reducción de la acción del viento y de forma respectivamente. Se consideró un tiempo de recurrencia de 50 años para lo cual el coeficiente de recurrencia $C t$ adopta el valor de 1.00. Para el caso del coeficiente de sitio $C s$, se clasifica el sitio como expuesto debido a que la edificación se ubica en una zona costera, de ahí que este coeficiente es igual a 1.10. Por otra parte, para el tipo de terreno abierto por estar en la línea de costa y una altura máxima de la edificación de $15.94 \mathrm{~m}$, el coeficiente de ráfaga $\mathrm{Cr}$ es 1.16, mientras que el coeficiente de altura Ch es 1.15. El coeficiente de reducción de la acción del viento $\mathrm{Cra}$ depende de la altura máxima y de la dimensión máxima de la superficie expuesta al viento, siendo esta última igual a $15.94 \mathrm{~m}$ correspondiente al panel de mayor dimensión del muro cortina, por lo que este coeficiente es 0.80 . Producto de la complejidad geométrica de la losa fueron considerados los coeficientes de forma definidos en NC-285 (2003), empleado para cubiertas tipo bóveda y similares por la forma, al considerarse semejante al caso 
de estudio. Atendiendo a que la curvatura de la losa varía en la dirección $\mathrm{Z}$, se dividió la losa en zonas con curvatura semejante para la consideración de los coeficientes de forma (Figura 5).

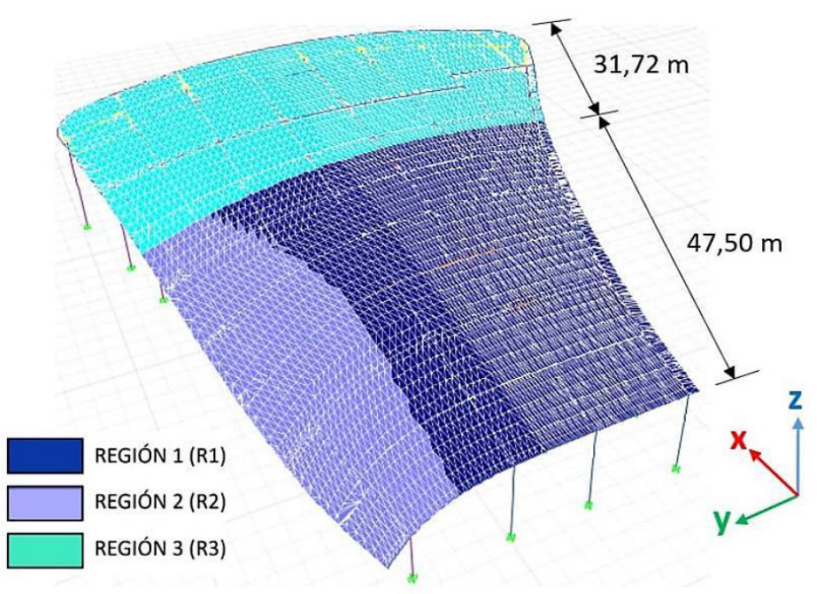

Figura 5: Regiones en las que fue dividida la losa para la consideración de los coeficientes de forma.

La carga de viento obtenida con la aplicación de (1) para cada una de las regiones consideradas, se presenta en la Figura 6.

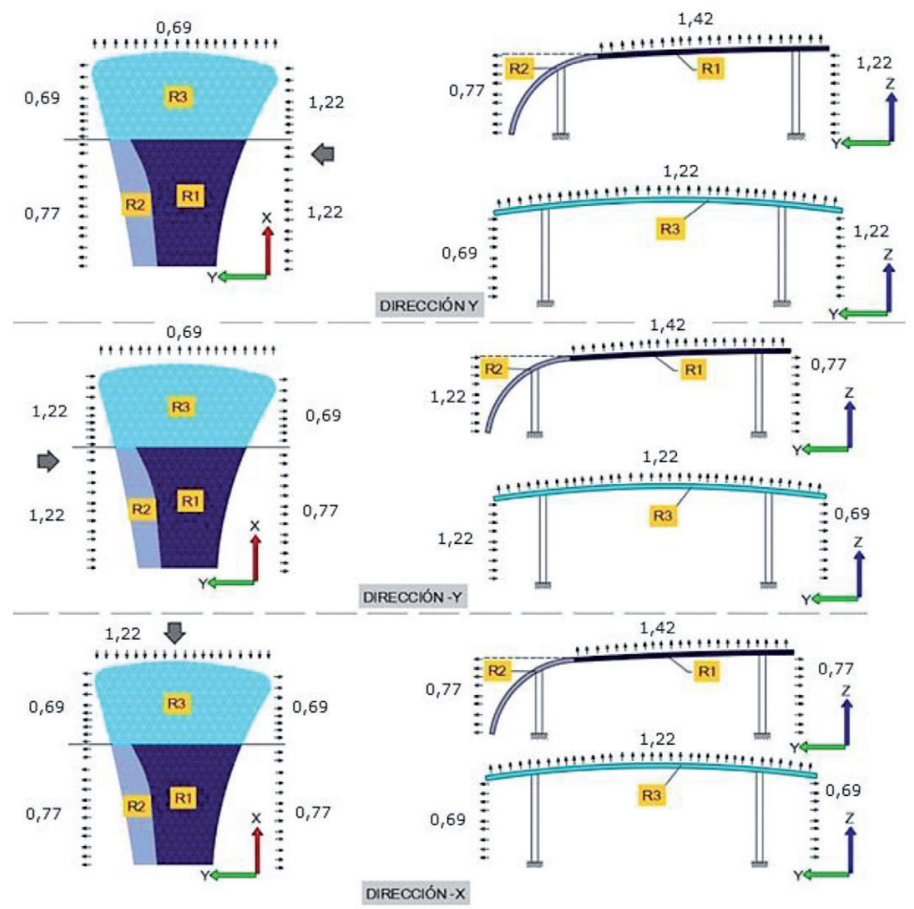

Figura 6: Carga de viento aplicada en $\mathrm{kN} / \mathrm{m}^{2}$. A1: dirección Y planta, A2: dirección Y elevación, B1: dirección -Y planta, B2: dirección $-\mathrm{Y}$ elevación, $\mathrm{C} 1$ : dirección $-\mathrm{X}$ planta, $\mathrm{C} 2$ : dirección -X elevación.

\section{Discretización del modelo analítico}

En el software ETABS se establece que el mallado de las losas inclinadas no será realizado automáticamente por el programa, sino que este quedará definido según el tamaño de losa modelado (CSI, 2017). En el proceso de modelación, la losa fue discretizada en múltiples elementos analíticos tipo shell de tres nodos, considerando como formulación de espesor el de placa delgada (thin shell) (CSI, 2017). El elemento de tres nodos permite modelar con más precisión la geometría curva de la losa y su unión con las vigas curvas (Figura 7). Además, se detectó errores al modelar elementos tipo shell de más de cuatro nodos.

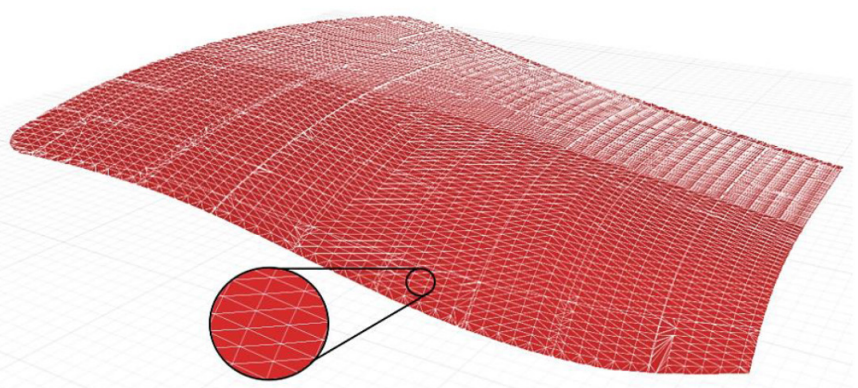

Figura 7: Discretización de la losa del modelo analítico

El elemento shell combina el análisis de dos tipos de comportamientos: comportamiento tipo membrana y placa-flexión (plate bending). El primero utiliza una formulación isoparamétrica que incluye una componente de rigidez traslacional en el plano y otra componente de rigidez rotacional, normal al plano del elemento. El segundo incluye en dos direcciones, fuera del plano, la componente rotacional de rigidez de la placa y la componente traslacional de rigidez en la dirección normal al plano del elemento (CSI, 2017).

El tamaño de los elementos triangulares es variable, dependiendo de la zona de losa modelada y de su interacción con las vigas. De forma general el promedio de tamaño de los triángulos es de $0.62 \mathrm{~m} \times 1.52 \mathrm{~m}$ (base $\mathrm{x}$ altura), para un área promedio de $0.47 \mathrm{~m}^{2}$. Considerando las dimensiones generales de la losa, se modeló un total de 12295 elementos para una densidad de 3.07 elementos por $\mathrm{m}^{2}$. La Figura 8 muestra el modelo final de la losa y el sistema de soporte vertical que la sustenta. 


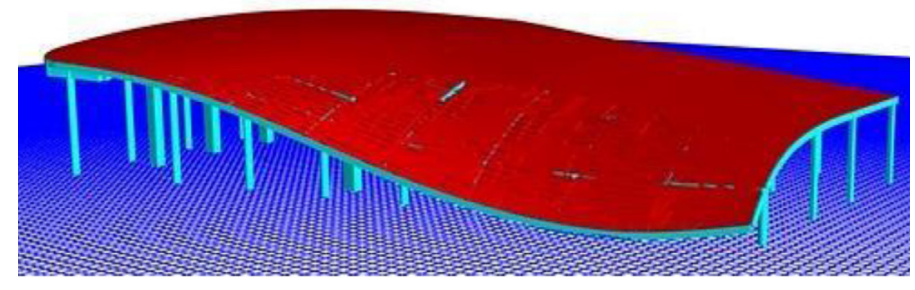

Figura 8: Modelo final de la losa y el sistema de soporte vertical que la sustenta.

\section{Formulación de espesor del elemento shell}

En el programa ETABS están disponibles dos tipos de formulación de espesor: placa gruesa (thick plate) y placa delgada (thin plate). La placa gruesa tiene en cuenta la formulación de Mindlin/Reissner, la cual incluye el efecto de la deformación por cortante transversal, mientras que la placa delgada (formulación de Kirchhoff) no lo tiene en cuenta. Las deformaciones por cortante tienden a ser significativas cuando el espesor de la losa es mayor que $1 / 10$ a $1 / 5$ de la luz de trabajo (CSI, 2017). También puede ser importante analizar la deformación por cortante en zonas donde haya cambios bruscos de espesor en la losa y cerca de huecos. Teniendo en cuenta las sugerencias para el uso de las formulaciones de espesor anteriores, en el caso particular de este trabajo, la formulación más adecuada es la de placa fina. Se considera esta formulación puesto que, de forma general, el espesor de la losa $(200 \mathrm{~mm})$ es inferior a la décima parte de la luz de trabajo. Además, no existen huecos en la losa ni cambios en el peralto. A pesar de que la formulación thick-plate tiende a ser más precisa, también tiende a ser más rígida que la de placas delgadas. Tampoco es recomendable utilizar esta formulación si no se trata de un mallado uniforme (CSI, 2017). En el caso del mallado de la losa que se analiza en este trabajo, este presenta distorsiones en algunas zonas producto de la forma compleja de la losa, por lo cual no se considera uniforme en toda su extensión.

No obstante, con la finalidad de comparar resultados de deformación absoluta de la estructura, se hizo un análisis considerando placas gruesas y delgadas. Se pudo establecer que la diferencia entre las deformaciones absolutas obtenidas por ambas formulaciones es ínfima (< $1 \%$ ), lo cual ratifica que la influencia de la deformación por cortante transversal en la deformación total de la estructura es pequeña por lo que puede despreciarse. Por ello, los mapas de isovalores de deformación obtenidos por ambas formulaciones son prácticamente idénticos. Más adelante, en la sección Deformación del epígrafe de Estado Límite de Servicio, se muestran los isovalores correspondientes a la formulación de placa delgada.

\section{Densidad de mallado}

Se analizó una densidad de mallado a partir del software SketchUp. La losa que se analiza en este trabajo no clasifica como losa tipo cáscara o lámina delgada de hormigón. Esto es porque, como se aprecia en el epígrafe Estado Límite Último, la losa está sometida a valores de momento elevados, lo cual no es la principal forma de trabajo de las cáscaras. Por otra parte, la losa presenta un entramado de vigas principales y secundarias cuyo peralto es considerable a diferencia de las láminas delgadas donde los elementos auxiliares son vigas de borde de poco peralto. El espesor de la losa (200 $\mathrm{mm})$ es muy superior a los espesores comunes en láminas delgadas (ACI-334, 2002). Como la losa de cubierta no es una cáscara delgada, el procedimiento de modelación se modifica con respecto al de este tipo de elementos y se dificulta mucho más.

El procedimiento para la obtención del mallado y el por qué solamente se emplea una densidad de malla se describe a continuación:

La curvatura de las vigas varía considerablemente y define en gran medida el comportamiento estructural de las mismas por lo que es indispensable que la modelación de su forma geométrica, cuya curvatura está en correspondencia con la de la losa, sea lo más exacta posible. Por esta razón, las vigas rigen la discretización de los elementos finitos que componen la losa de cubierta.

1. Para lograr esto se parte del modelo arquitectónico en SketchUp, el cual provee la forma exacta de la losa de cubierta deseada por los arquitectos, sin embargo, no posee las vigas; estas son añadidas minuciosamente en el propio SketchUp, a partir de utilizar una plantilla de AutoCAD como guía, que provee las proyecciones de las vigas en planta. Las vigas se dibujan a través de la losa, a la cual previamente se le ha descubierto el mallado en SketchUp, en este programa el mallado de los elementos por defecto es siempre triangular. La representación de las vigas se realiza mediante 
segmentos cuya longitud está definida por los elementos de tres nodos de la losa.

2. Una vez modeladas las vigas se exporta el modelo a AutoCAD para crear una plantilla tridimensional que se utilizará como base para el modelado en ETABS.

3. Con la plantilla tridimensional en ETABS se procede a modelar cada elemento de tres nodos que conforman la losa de forma individual, uno a uno, haciendo un total de 12295 elementos.

En ETABS el mallado de losas curvas está definido por las dimensiones de los elementos individuales modelados (CSI, 2017), o sea, no se debe asignar un mallado automático puesto que genera errores en el cálculo. Por lo que si se deseara hacer un mallado con elementos de cuatro nodos sería necesario modelar toda la estructura desde cero, determinando primero la coordenadas $(x, y, z)$ de cada punto de la malla, que debe ser compatible con las coordenadas de cada punto de las vigas curvas, y por tanto, no se podría emplear el modelo de SketchUp ya que este solo proporciona un mallado de tres nodos. Por otra parte, si se desea realizar un análisis de mallado óptimo para tres o cuatro nodos es necesario modelar nuevamente la estructura desde el inicio, repitiendo todos los pasos anteriores, y no es posible simplemente asignar un mallado más denso, de forma automática, como usualmente se hace en este tipo de análisis. Por todo esto, se utilizan elementos de tres nodos, ya que aproximan mejor la forma geométrica de la losa y son obtenidos directamente del modelo arquitectónico.

\section{Resultados}

El diseño estructural de la losa de cubierta se realizó a partir de lo establecido en NC-207 (2019). Se verificó el cumplimiento de los Estados Límites Último y de Servicio. En el caso del Estado Límite de Servicio, se comprobó el cumplimiento de la deformación permisible de la losa y todas las vigas y se verificó el cumplimiento del ancho de fisura máximo permisible atendiendo a la agresividad ambiental (NC-207, 2019).

\section{Estado Límite Último}

Se verificó que la combinación pésima de cargas fue $1.2 D+1.6 L_{\mathrm{c}}$, siendo $D$ la carga muerta y $L_{\mathrm{c}}$ la carga viva de cubierta. Con el fin de realizar un diseño eficiente se dividió la losa por momentos actuantes de similar valor a partir de las solicitaciones obtenidas del programa ETABS (Figura 9).

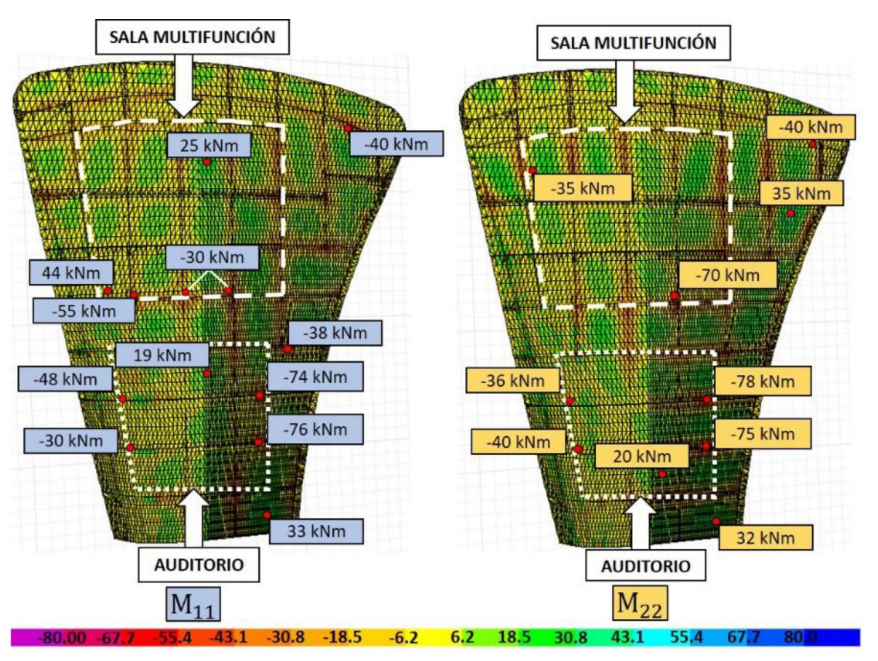

Figura 9: Mapas de isovalores de los máximos momentos flectores reportados. Combinación de cargas $1.2 D+1.6 L_{c}$

El cálculo de refuerzo se realizó siguiendo la metodología de diseño establecida en ACI-318 (2019) y NC-207 (2019) garantizando que el momento último actuante $M_{u}$ sea menor o igual que el momento nominal de la sección afectado por un coeficiente reductor de la capacidad nominal $\varnothing,\left(M_{u} \leq\right.$ $\left.\varnothing M_{n}\right)$. Las expresiones (2), (3) y (4) fueron utilizadas para una sección rectangular de hormigón (ACI-318, 2019; NC-207, 2019).

$$
\begin{aligned}
& M_{u}=\varnothing\left[0.85 f_{c}^{\prime} a b\left(d-\frac{a}{2}\right)\right] \\
& A_{s}=\frac{0.85 f_{c}^{\prime} a b}{f_{y}} \\
& a=\beta_{1} c
\end{aligned}
$$

donde $A_{s}$ es el área de acero en tracción, $f_{y}$ es la tensión de fluencia del acero ordinario, $f_{c}^{\prime}$ es la resistencia característica a compresión del hormigón a los 28 días, $d$ es la distancia de la fibra extrema en compresión al centroide del acero en tracción, $b$ es el ancho de la sección de hormigón, $\alpha$ es la altura del bloque rectangular equivalente de esfuerzos, $\beta$ es el factor que depende de la resistencia a compresión del hormigón y $c$ es la profundidad de la línea neutra. La Tabla 1 resume los resultados del cálculo del refuerzo de la losa. 
Tabla 1: Refuerzo por resistencia

\begin{tabular}{|c|c|c|c|c|c|}
\hline \multirow{2}{*}{$\begin{array}{c}\text { Momento } \\
\text { último de } \\
\text { diseño } M_{\mathrm{u}}, \\
\mathrm{kNm}\end{array}$} & $\begin{array}{c}\text { Diámetro, } \\
\mathrm{mm}\end{array}$ & $\begin{array}{c}\text { Área de } \\
\text { acero, } \\
\mathrm{cm}^{2}\end{array}$ & $\begin{array}{c}\text { Espacia- } \\
\text { miento, } \\
\mathrm{mm}\end{array}$ & $\begin{array}{c}\text { Área de } \\
\text { acero } \\
\text { mínima } \\
\mathrm{cm}^{2}\end{array}$ & $\begin{array}{c}\text { Espacia- } \\
\text { miento } \\
\text { máximo, } \\
\mathrm{mm}\end{array}$ \\
\hline \pm 30 & 16 & 7.65 & 260 & & \\
\hline 32 & 16 & 8.29 & 240 & & \\
\hline \pm 38 & 16 & 9.95 & 200 & & \multirow{2}{*}{2.45} \\
\hline-40 & 16 & 10.47 & 190 & \\
\hline 44 & 16 & 11.70 & 170 & & \\
\hline-48 & 16 & 13.27 & 150 & & \\
\hline-77 & 16 & 22.11 & 90 & & \\
\hline
\end{tabular}

\section{Estado Límite de Servicio}

\section{Fisuración}

En este proyecto resultó imprescindible el chequeo del cumplimiento de la abertura de fisura permisible debido a la agresividad del medio, la cual contribuye al incremento acelerado de la corrosión de las barras de refuerzo de la losa. El ancho de fisura $\alpha_{f}$ se determinó a partir del enfoque de Frosch. Reconocido por el Comité ACI 224R (2001), este modelo de predicción de las fisuras es adoptado como referencia para las normativas del ACI a partir de 1999. La abertura de fisura se determinó por la expresión (5).

$$
a_{f}=2 \frac{f_{s}}{E_{s}} \beta \sqrt{d_{c}^{2}+\left(\frac{s}{2}\right)^{2}}
$$

donde $f_{s}$ es la tensión de trabajo del acero en $\mathrm{MPa}, E_{s}$ el módulo de deformación del acero en $\mathrm{MPa}, d_{c}$ es la distancia del borde de la fibra más traccionada al centroide de la barra más cercana a ella en mm, $s$ es la separación entre los centros de las barras de la armadura principal por flexión más cercana a la cara más traccionada en mm y $\beta$ es denominado factor de profundidad, el cual puede ser calculado aproximadamente por la expresión (6) (Hernández y Hernández, 2010).

$$
\beta=1+0.0031 d_{c}
$$

Al analizar la fisuración en la losa con el refuerzo calculado por flexión se determinó que no se cumplía con la abertura de fisura permisible, razón por la que fue necesario rediseñar el refuerzo en función del Estado Límite de Fisuración. En la Tabla 2 se presentan los resultados del cálculo de la abertura de fisura según ACI 224R (2001), donde $a_{\mathrm{k}}$ y $a_{\mathrm{f}}$ corresponden a las aberturas de fisura permisible $\mathrm{y}$ calculada respectivamente. Es importante resaltar que, debido a la agresividad del ambiente, se consideró que la losa tendrá una membrana protectora, por lo que según ACI 224R (2001), el ancho de fisura permisible será de $0.41 \mathrm{~mm}$. La membrana protectora no modifica las características de fisuración de la losa. Es un requisito para poder adoptar una abertura de fisura permisible de 0.41 $\mathrm{mm}$ y es una recomendación para el proyecto. ACI-224R (2001) no especifica las características de la membrana protectora, sino que se presenta una guía para anchos de fisura máximos para diferentes condiciones de exposición, entre las que se encuentra membrana protectora. En este punto del proyecto no se cuenta todavía con un proveedor de membrana protectora por lo que no se mencionan características específicas de estas. No obstante, existen numerosas compañías dedicadas a la distribución de productos de esta categoría. Por ejemplo, una amplia gama de productos especiales para la impemeabilización y protección de cubiertas, como imprimaciones bituminosas, membranas líquidas, etc. Una de estas categorías de productos son los recubrimientos de alta reflectancia, entre las características principales está la reducción de la temperatura de la superficie, incluso si se expone a luz solar fuerte por lo que contribuye al ahorro de energía. Dentro de esta categoría se encuentran dos tipos de productos: el primero de estos es una membrana líquida reforzado con fibra a base de agua, formulada con resinas sintéticas especiales; la segunda es una pintura protectora a base de solvente, con alta reflectancia solar que califica, además, como una película protectora.

\begin{tabular}{|c|c|c|c|c|}
\hline Solicitación & \multicolumn{4}{|c|}{ Refuerzo por fisuración } \\
\hline $\begin{array}{c}\text { Momento de } \\
\text { servicio } M_{\mathrm{k}}, \mathrm{kNm}\end{array}$ & $\varnothing, \mathrm{mm}$ & (a) mm & $a_{\mathrm{k}}, \mathrm{mm}$ & $a_{\mathrm{f}}, \mathrm{mm}$ \\
\hline \pm 24 & \multirow{7}{*}{16} & 100 & 0.41 & 0.26 \\
\hline 26 & & 100 & 0.41 & 0.26 \\
\hline \pm 30 & & 100 & 0.41 & 0.26 \\
\hline-32 & & 100 & 0.41 & 0.26 \\
\hline 36 & & 100 & 0.41 & 0.26 \\
\hline-39 & & 100 & 0.41 & 0.26 \\
\hline-62 & & 70 & 0.41 & 0.24 \\
\hline
\end{tabular}

Tabla 2: Refuerzo por fisuración 
Al comparar los resultados presentados en la Tabla 1 con los que se muestran en la Tabla 2 se evidencia que en el diseño del refuerzo predomina el Estado Límite de Fisuración disminuyendo el espaciamiento de las barras en relación con el obtenido para el Estado Límite Último.

\section{Deformación}

A partir del mapa de isovalores de deformación absoluta $\Delta_{a b c}$ de la estructura, obtenido de ETABS, se localizaron las zonas de la losa con mayor deformación y se determinó, el valor de flecha relativa total en estos puntos (Figura 10).

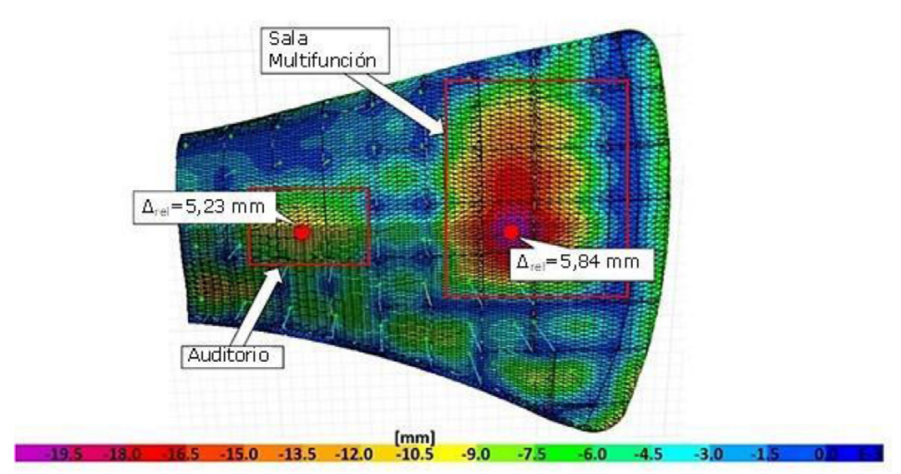

Figura 10: Mapa de isovalores de deformación relativa total de la losa. Combinación: $D+L_{\mathrm{c}}$

En este caso se verificó que las mayores deformaciones se encuentran en el salón multifunción y en el auditorio. La flecha máxima permisible según NC-207 (2019) es L/480, correspondiente a losas y vigas que se encuentran vinculados a elementos no estructurales susceptibles de ser dañados por flechas excesivas, donde $L$ corresponde a la luz de cálculo. Debido a que el hormigón se deforma diferidamente bajo la acción de cargas sostenidas (Hernández y Hernández, 2010), es estrictamente necesario que, adicionalmente a la flecha de corta duración se considere una flecha diferida. En el análisis de la flecha con el programa ETABS, estos aspectos fueron considerados. Para validar los resultados obtenidos se realizó la comparación con el procedimiento clásico aproximado de cálculo de flechas en losas que trabajan en una dirección. La flecha total se determina a partir de las expresiones (7) y (8) (NC-207, 2019).

$$
\begin{aligned}
& \Delta_{t o t}=\Delta_{t e}+\lambda_{\infty} \Delta_{p t l d} \\
& \Delta_{t e}=\Delta_{p t}-\Delta_{p t l d}
\end{aligned}
$$

donde $\Delta_{t o t}$ es la flecha total, $\Delta_{t e}$ es la flecha instantánea de corta duración, $\Delta_{p t l d}$ es la flecha instantánea producto de la carga permanente y la carga de uso de larga duración (25\% de la carga de uso de servicio, establecido por proyecto), $\Delta_{p t}$ es la flecha instantánea producto de la carga permanente y de uso de servicio y $\lambda_{\infty}$ es un coeficiente que toma en cuenta la acción de las cargas sostenidas de forma indefinida, es decir, que la carga de uso de larga duración se consideró que actúa de forma indefinida en la losa; dicho coeficiente se determina mediante la expresión (9) (NC-207, 2019).

$$
\lambda_{\infty}=\frac{T}{1+50 \rho^{\prime}}
$$

En la expresión (9), $T$ es un factor dependiente del tiempo de permanencia de la carga, que en este caso es 2 , correspondiente a 5 años o más, y $\rho^{\prime}$ es la cuantía geométrica del refuerzo en compresión. Para obtener las flechas instantáneas de cada elemento se empleó la rigidez efectiva $E_{c}^{\prime} I_{e}$, donde $E_{c}^{\prime}$ es el módulo de deformación del hormigón e $I_{e}$ es la inercia efectiva de la sección fisurada y se calculan por medio de la expresión (10):

$$
I_{e}=I_{c r}+\left(\frac{M_{c r}}{M}\right)^{3}\left(I_{h}-I_{c r}\right) \leq I_{h}
$$

Siendo $I_{c r}$ la inercia de la sección fisurada, $M_{c r}$ el momento de fisuración, $M$ el momento según la flecha instantánea analizada, e $I_{h}$ la inercia de la sección homogeneizada. La inercia de la sección fisurada se determina a partir de la expresión (11) donde $b, x, d, n$ y $A_{s}^{\prime}$ corresponden al ancho de la sección de hormigón, la profundidad de la línea neutra en servicio, el peralto efectivo de la sección, el módulo de equivalencia (expresión (12)) y el área de acero en compresión, respectivamente.

$$
\begin{aligned}
& I_{c r}=\frac{b x^{3}}{3}+n A_{s}(d-x)^{2}+(n-1) A_{s}^{\prime}\left(x-d^{\prime}\right)^{2} \\
& n=\frac{E_{s}}{E_{c}^{\prime}}
\end{aligned}
$$

Las flechas totales calculadas son $5.76 \mathrm{~mm}$ y $6.10 \mathrm{~mm}$ para el salón multifunción y el auditorio, respectivamente, valores muy similares a los obtenidos mediante ETABS, lo que valida estos últimos, cumpliéndose además con la flecha máxima permisible, que tanto para el salón multifunción como para el auditorio es $16.77 \mathrm{~mm}$. 
Los resultados del análisis de la máxima deformación en las vigas se resumen en la Tabla 3 , las cuales corresponden al voladizo (Figura 11), donde las luces abarcan desde 3.14 $\mathrm{m}$ hasta $10.23 \mathrm{~m}$.

Tabla 3: Comprobación de la deformación en las vigas. Máximos valores reportados para la combinación: $D+L_{\mathrm{c}}$

\begin{tabular}{|c|c|c|c|c|c|c|}
\hline Elemento & $\begin{array}{c}\text { Sección, } \\
\mathrm{mm}\end{array}$ & $\begin{array}{c}\Delta \text { dif, } \\
\mathrm{mm}\end{array}$ & $\begin{array}{c}\text { Luz, } \\
\mathrm{mm}\end{array}$ & $\begin{array}{c}\Delta \text { perm, } \\
\mathrm{mm}\end{array}$ & $\begin{array}{c}\text { Cumpli- } \\
\text { miento }\end{array}$ & $\begin{array}{c}\text { Diferencia, } \\
\mathrm{mm}\end{array}$ \\
\hline V121 & $1800 \mathrm{X} 600$ & 0.89 & 3136 & 6.53 & cumple & 5.65 \\
\hline V122 & $1800 \mathrm{X} 600$ & 11.38 & 4376 & 9.12 & $\begin{array}{c}\text { no } \\
\text { cumple }\end{array}$ & -2.26 \\
\hline V123 & $1800 \times 600$ & 10.85 & 4340 & 9.04 & $\begin{array}{c}\text { no } \\
\text { cumple }\end{array}$ & -1.81 \\
\hline V124 & $1800 \times 600$ & 10.25 & 4326 & 9.01 & $\begin{array}{c}\text { no } \\
\text { cumple }\end{array}$ & -1.24 \\
\hline V125 & $1800 \times 600$ & 9.95 & 4345 & 9.05 & $\begin{array}{c}\text { no } \\
\text { cumple }\end{array}$ & -0.90 \\
\hline V126 & $1800 \times 600$ & 11.10 & 4390 & 9.15 & $\begin{array}{c}\text { no } \\
\text { cumple }\end{array}$ & -1.95 \\
\hline V127 & $1800 \times 600$ & 10.50 & 4460 & 9.29 & $\begin{array}{c}\text { no } \\
\text { cumple }\end{array}$ & -1.21 \\
\hline V128 & $1800 \times 600$ & 15.83 & 5500 & 11.46 & $\begin{array}{c}\text { no } \\
\text { cumple }\end{array}$ & -4.37 \\
\hline V141 & $1800 \times 600$ & 15.75 & 10230 & 21.31 & $\begin{array}{c}\text { cumple } \\
5.56\end{array}$ \\
\hline
\end{tabular}

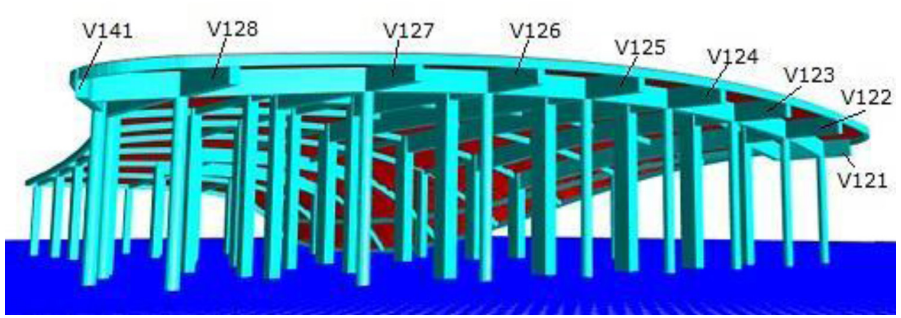

Figura 11: Vigas con mayor deformación

Como se puede apreciar en la Tabla 3, existen vigas que no cumplen con la deformación máxima permisible. Las diferencias entre las flechas calculadas y las permisibles oscilan entre $0.9 \mathrm{~mm}$ y $4.37 \mathrm{~mm}$. Según NC-207 (2019), la flecha máxima puede ser excedida siempre que se garantice que los elementos no estructurales susceptibles no sean dañados. Lo anterior puede lograrse a partir de juntas de neopreno.

\section{Verificación de requisitos de resistencia al fuego}

La resistencia estructural al fuego es un tema importante que debe tenerse en cuenta durante el proceso de diseño de una edificación para minimizar la posibilidad de fallas estructurales inducidas en caso de un incendio. Las propiedades físicas y químicas de los materiales se degradan cuando estos se ven expuestos a elevadas temperaturas, provocando reducción de la resistencia, el módulo de elasticidad y la rigidez de los elementos estructurales y, por consiguiente, un fallo localizado o el colapso progresivo de los mismos. Es por ello que para garantizar que la integridad estructural se mantenga durante un período de tiempo adecuado, los elementos deben diseñarse para satisfacer, además de los requisitos estructurales definidos en los códigos de construcción, los requisitos adecuados de resistencia al fuego en caso de incendio.

En el diseño de estructuras en situación de incendio, debe verificarse que la resistencia de la estructura (o parte de ella) sea superior a la severidad del incendio a la que se expone. Este chequeo puede ser realizado en el dominio de la temperatura, de la resistencia o del tiempo. En el dominio de la temperatura debe garantizarse que la temperatura alcanzada por los materiales sea inferior a su temperatura crítica (temperatura que causa el colapso en el elemento estructural) mientras que el dominio de la resistencia se garantiza a partir del cumplimiento de los estados límites (expresión (14)):

$$
S_{u, \theta} \leq S_{n, \theta}
$$

Donde $S_{u, \theta}$ es el valor de cálculo de los esfuerzos actuantes en situación de incendio y $S_{n, \theta}$ es el valor de cálculo de los esfuerzos resistentes en situación de incendio. En el dominio del tiempo, debe cumplirse que el Tiempo de Resistencia al Fuego (TRF) de una estructura sea mayor o a lo sumo igual al Tiempo Requerido de Resistencia al Fuego (TRRF). El Tiempo de Resistencia al Fuego TRF se define como el tiempo máximo de resistencia, considerado como el periodo entre el inicio del siniestro y el instante del colapso de la estructura (Costa, 2008). El Tiempo Requerido de Resistencia al Fuego TRRF que es el tiempo mínimo que vigas, losas o columnas deben resistir cuando son sometidas al incendio estándar (ABNT NBR 14432, 2001), y es dependiente del tipo de ocupación o uso y la altura de la edificación.

Los códigos de diseño que abordan el tema (ACI 216, 1997; ABTN NBR 15200, 2012; EN 1992-1-2, 2004) proponen métodos simplificados, gráficos y/o tabulares, 
que aseguran tiempos de resistencia al fuego para los elementos de hormigón armado, a partir de establecer dimensiones y recubrimientos mínimos. De igual forma, documentos como la normativa francesa (NF EN 19911-2, 2005), española (DB-SI, 2010) y brasileña (NBR 14432, 2001), establecen tiempos requeridos de resistencia al fuego según el uso y altura de la edificación. Tomando como referencia los documentos antes citados, para la losa diseñada, perteneciente a un hotel de altura máxima 15.94 $\mathrm{m}$, el tiempo máximo de resistencia requerida al fuego TRRF es igual a $90 \mathrm{~min}$.

Según el método tabular simplificado propuesto en el EN 1992-1-2 (2004), para losas de hormigón armado trabajando en dos direcciones, con espesor mínimo de $150 \mathrm{~mm}$ ((200 $\mathrm{mm}>150 \mathrm{~mm}$ ) y un recubrimiento mínimo (profundidad medida al centroide de la barra) de $40 \mathrm{~mm}$ (48 mm $>40$ $\mathrm{mm}$ ), la resistencia al fuego queda garantizada durante un tiempo de $180 \mathrm{~min}$.

A partir de estos resultados se concluye que el diseño propuesto para la losa puede ser considerado satisfactorio, garantizándose un tiempo de resistencia al fuego para el elemento tal que, TRF $\geq \operatorname{TRRF}(180 \mathrm{~min} \geq 90 \mathrm{~min})$.

\section{Construcción}

El volumen total de hormigón de la losa es $802 \mathrm{~m}^{3}$, mientras que el peso total del refuerzo de acero es $222 \mathrm{t}$, por lo que el índice de consumo del acero será $277 \mathrm{~kg} / \mathrm{m}^{3}$. El contenido mínimo de cemento debe ser de $350 \mathrm{~kg} / \mathrm{m}^{3}$ debido a la elevada agresividad del ambiente (NC-120, 2004; NC207, 2019). La curvatura que caracteriza esta losa trae como consecuencia que el procedimiento de construcción sea similar al de una lámina o cáscara de hormigón. El tamaño máximo del árido será $19.0 \mathrm{~mm}$ cumpliendo con lo enunciado en NC-207 (2019) que establece que el tamaño del agregado no debe exceder la menor de las siguientes distancias: 0.33 del espesor de la losa, 0.75 del espaciamiento mínimo libre entre las barras de refuerzo y 0.90 de la separación entre barras de camadas contiguas. El tamaño máximo de árido escogido corresponde al valor ajustado según el tamaño normado de los áridos nacionales (NC-251, 2013). La forma de distribución del refuerzo puede ser apreciada en la Figura 12.
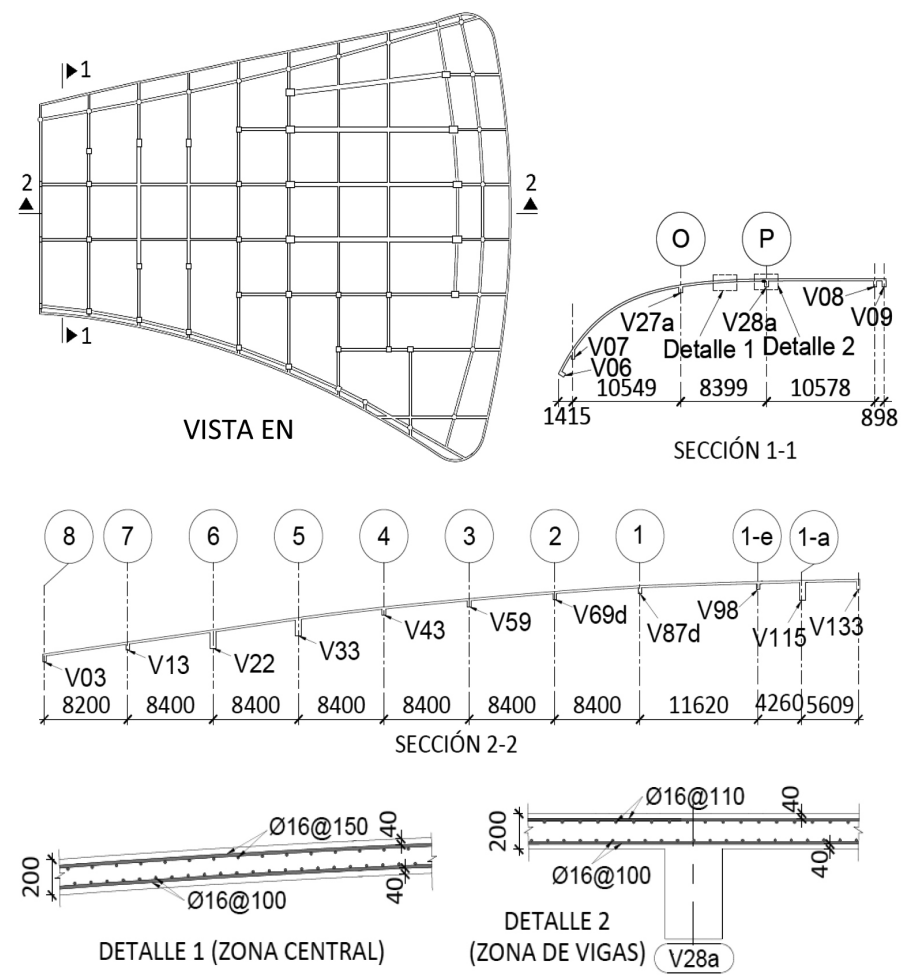

Figura 12: Distribución del refuerzo en la losa

\section{Conclusiones}

El desarrollo de un modelo computacional con el empleo del programa ETABS para el análisis y diseño estructural de la losa con forma geométrica compleja permitió obtener resultados precisos de solicitaciones y deformaciones. Como resultado del análisis se pudo apreciar que las zonas de la losa más solicitadas corresponden a los locales del auditorio y la sala polivalente, mientras que las vigas con mayor deformación se encuentran en los voladizos, los que requieren de una solución que garantice que los elementos no estructurales no sufran daño. Por otra parte, el Estado Límite de Fisuración impuso un incremento del refuerzo de la losa con respecto al refuerzo obtenido por resistencia, siendo el refuerzo para el momento máximo inferior en la zona traccionada de 16 mm @ 100 mm, y para el momento máximo superior en la zona traccionada de 16 mm @ 70 $\mathrm{mm}$. Se comprobó además que el espesor dado a la losa garantiza un tiempo de resistencia al fuego mayor que el requerido. 


\section{Referencias}

ABNT NBR 14432 (2001). Exigências de resistência ao fogo de elementos construtivos de edificações - Procedimento. Comitê Brasileiro da Segurança contra Incêndio, Associação Brasileira de Normas Técnicas, Brasil

ABTN NBR 15200 (2012). Projeto de estruturas de concreto em situação de incêndio. Comitê Brasileiro da Construção Civil, Associação Brasileira de Normas Técnicas, Brasil

ACI-334 (2002). Concrete shell structures. Practice and Commentary. American Concrete Institute, Farmington Hills, MI, USA

ACI-224R (2001). Control of cracking in concrete structures. American Concrete Institute, Farmington Hills, MI, USA

ACI-318 (2019). Building code requirements for structural concrete. American Concrete Institute, Farmington Hills, MI, USA

ACI 216 (1997). Code requirements for determining fire resistance of concrete and masonry construction assemblies. American Concrete Institute, Farmington Hills, MI, USA

Costa, C.N. (2008). Dimensionamento de elementos de concreto armado em situação de incêndio. Tese de Doutorado, Universidade de São Paulo, Brasil

CSI (2017). ETABS v17. Computers \& Structures, Inc. CSI. Structural and earthquake engineering software. USA

DB-SI (2010). Documento básico de seguridad en caso de Incendio. Código Técnico de la Edificación Ministerio de Fomento, Dirección General de la Arquitectura, Vivienda y Suelo. España

EN 1992-1-2 (2004). Eurocode 2: Design of concrete structures. Part 1-2: General rules - Structural fire design. European Committee for Standardization, Brussels, Belgium

Genikomsou, A. and Polak, A.M. (2017). Finite element simulation of concrete slabs with various placement and amount of shear bolts. Procedia Engineering 193, 313-320

Gherbi, A., Dahmani, L. and Boudjemia, A. (2018). Study on two way reinforced concrete slab using ANSYS with different boundary conditions and loading. Engineering and Technology International Journal of Civil and Environmental Engineering 12(12), 1151-1156
González, Ó. y Robles, F. (2005). Aspectos fundamentales del concreto reforzado. Editorial Limusa, México

Hernández, J.J. y Hernández, J.A. (2010). Hormigón estructural. Diseño por estados límites. Vol. 1 y 2. La Habana, Cuba

Jiménez, P., García, A. y Morán, F. (2000). Hormigón armado. Editorial Gustavo Gili, México

McCormac, J.C. y Brown, R.H. (2011). Diseño de concreto reforzado. Alfaomega, México

NC-120 (2004). Hormigón hidráulico. Especificaciones. Oficina Nacional de Normalización, Cuba

NC-207 (2019). Requisitos generales para el diseño y construcción de estructuras de hormigón. Oficina Nacional de Normalización, Cuba

NC-250 (2005). Requisitos de durabilidad para la construcción de edificaciones y obras civiles de hormigón estructural. Oficina Nacional de Normalización, Cuba

NC-251 (2013). Áridos para hormigones hidráulicos. Requisitos. Oficina Nacional de Normalización, Cuba

NC-283 (2003). Densidad de materiales naturales, artificiales y de elementos de construcción como carga de diseño. Oficina Nacional de Normalización, Cuba

NC-284 (2003). Edificaciones. Carga de uso. Oficina Nacional de Normalización, Cuba

NC-285 (2003). Carga de viento. Método de cálculo. Oficina Nacional de Normalización, Cuba

NF EN 1991-1-2 (2005). Eurocode 1: Actions sur les structures. Partie 1-2: Actions générales - Actions sur les structures exposées au feu. Association Francaise de Normalisation AFNOR. Paris, France

Rasoul, Z.M.R.A. and Taher, H.M.A.M. (2019). Accuracy of concrete strength prediction behavior in simulating punching shear behavior of flat slab using finite element approach in Abaqus. Periodicals of Engineering and Natural Sciences 7(4), 1933-1949

Salguero, F., Romero, S., Melgar, S.G., Prat, F. y Moreno, F. (2013). Las curvas tensión-deformación de hormigones ensayados bajo compresión uniaxial monotónica: una revisión sistemática. Informes de la Construcción 65(529), 41-54 